\title{
Tangence
}

\section{Lecture sociocritique d'un fait divers}

\section{Edmond Cros}

Numéro 37, septembre 1992

Autopsie du fait divers

URI : https://id.erudit.org/iderudit/025727ar

DOI : https://doi.org/10.7202/025727ar

Aller au sommaire du numéro

Éditeur(s)

Tangence

ISSN

1189-4563 (imprimé)

1710-0305 (numérique)

Découvrir la revue

Citer cet article

Cros, E. (1992). Lecture sociocritique d'un fait divers. Tangence, (37), 81-86.

https://doi.org/10.7202/025727ar d'utilisation que vous pouvez consulter en ligne.

https://apropos.erudit.org/fr/usagers/politique-dutilisation/ 


\section{Lecture sociocritique d'un fait divers}

\section{Edmond Cros*}

Ce qui retient en premier l'attention, c'est bien le montage de ce texte de faits divers: une photo accompagnée d'une légende, un gros titre, un commentaire bref du titre en caractère gras, une série de quatre très courts paragraphes censés apporter des précisions. Au total, quatre caractères différents chargés de hiérarchiser l'information. C'est ainsi que se détachent les éléments suivants par ordre d'importance donné dans le montage: famille massacrée par un des enfants adolescent, lequel doit être jugé comme adulte. D'entrée de jeu s'inscrit, on le voit, en arrière-plan de ce fait divers, un discours implicite, un non-dit qui implique un débat sur la conception de la justice. Faut-il appliquer à un mineur la rigueur de la justice? Et pourquoi ne l'appliquerait-on pas? Voyez le cas de figure précisément évoqué ici d'un acte particulièrement sauvage et révoltant. De telle sorte que la fonction sociale que l'on fait jouer ici au sait divers" ne peut être comprise que par rapport à un discours qui est contradictoire à celui qui se donne à voir dans cette dépêche de l'agence de presse et qui est un discours que, pour faire vite, je qualifierai de libéral et d'humaniste. C'est en fait à ce discours libéral que répond le texte qui nous intéresse.

Au service de cette réponse, plusieurs procédés: le jeu des contradictions, la composition et la hiérarchie de l'information, le système d'énonciation. La photo est l'image du bonbeur familial; les cinq personnages sont souriants, incarnant ainsi de façon exemplaire une des valeurs essentielles des sociétés nord-américaines. À y regarder de plus près, cependant, la disposition des actants est singulière. Dans la partie inférieure s'ordonne un schéma qu'on peut considérer comme traditionnel dans ce genre de représentation: père, mère, enfant. La partie supérieure tout au contraire est l'espace de la perturbation: le couple frère-sœur s'intercale entre les deux parents qu'il repousse sur les bords et marginalise, faisant apparaître un autre triangle qui est l'image

C.E.R.S, Institut international de sociocritique, Université Paul Valéry, Montpellier. 
82

pervertie du premier. On remarquera la parfaite homologie qui se construit dans les permutations fille/mère, fils/père, l'élément masculin se trouvant, dans les deux cas, légèrement surélevé; cette configuration convoque la représentation de rapports incestueux, d'une part entre le fils et la mère/fille, et de l'autre entre la fille et le fils/père. Une telle indiscrimination, qui suppose la confusion des générations, relève de l'abolition de la loi du père, abolition représentée ici par l'effacement du chef de famille. On ne s'étonne pas, dans ces conditions, que le fils dicte les interdits, dans ce cas ne pas écouter certaines cassettes, et que leur éventuelle transgression ait pu entraîner un châtiment (assassinat), reconstitutions des mobiles du meurtre que le montage même du texte nous invite à faire; l'interrogatoire en effet s'est orienté vers les mobiles de ce meurtre et les seuls éléments qui nous en parviennent font état d'un interdit posé par le fils qui aurait été transgressé par le père. Le dernier paragraphe du texte nous servira de légende dans la mesure où il commente parfaitement notre lecture sémiotique: l'adolescent *avait des problèmes avec ses parents [...] avait des problèmes avec son père *.

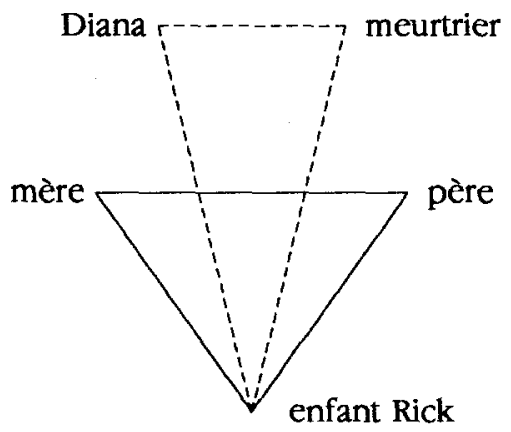

Mais l'adolescent peut être perçu aussi bien comme exclu de l'espace familial que comme dominateur. Son exclusion est d'ailleurs retranscrite dans le texte; d'abord dans la légende de la photo où le démonstratif "celui qui aperçoit "admet deux interprétations dans la mesure où il peut soit renvoyer à l'antécédent "membres de cette famille. (construction en ce cas relativement incorrecte), soit exprimer l'indétermination. $\mathrm{Et}$, de ce fait, l'adolescent est le seul à ne pas être désigné par son nom. Porte-t-il le 
même nom que son père? Son père est-il son père? Les contours de la famille restent en effet flous puisque, d'un côté, la photo implique que toute la famille soit réunie, tandis que le texte suggère une famille plus nombreuse: *un de ses frères, une des ses sceurs *. La photo cache ainsi plus qu'elle ne révèle: elle cache essentiellement des tensions et des relations; elle sacrifie à l'apparence, à la représentation idéologique du bonheur qui impose le refoulement de ce qui n'est pas acceptable par une société puritaine.

La photo suffirait à commenter le titre: «Une famille massacrée à la hache ", du moins dans le cadre d'une analyse sémiotique qui permettrait d'établir une corrélation entre le faux bonheur du passé et ce qu'a de monstrueux l'épisode du présent, en expliquant ce présent tragique par les graves fractures qui ont affecté la cellule familiale. À ce niveau, il n'y a pas de véritable contradiction. C'est bien pourtant sur la contradiction bonheur/ massacre que joue le montage journalistique et, par conséquent, sur les menaces qui planent sur les familles nord-américaines. Le jeu des caractères et de la représentation est destiné à accrocher le regard, à retenir l'attention, à faciliter l'assimilation et la mémorisation de cette corrélation dont on vient de voir qu'elle est essentiellement de nature idéologique: bonbeur/danger.

Bonheur —- Photo

Danger ——Titre

Composition et hiérarchie L'énonciation de l'information et l'imbrication des voix

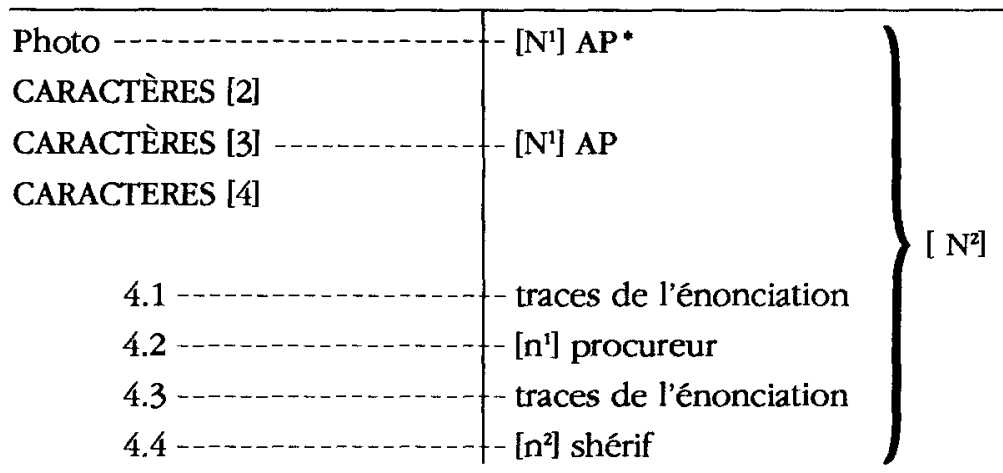

* AP désigne ici l'agence de presse. 
84

Le montage ne s'arrête pas là: il est le fait d'un narrateur [ $\left.\mathrm{N}^{2}\right]$, lequel reprend l'information d'un narrateur $\left[\mathrm{N}^{1}\right]$ qui s'autodésigne dans l'indéfini réitéré d'AP, c'est-à-dire d'une institution journalistique, censée ne donner que des informations objectives. Mais ce narrateur [ $\left.\mathrm{N}^{1}\right]$ reprend les propos de plusieurs narrateurs secondaires [n]: le procureur du comté, la police, le shérif du comté, soit explicitement, soit indirectement, semble-t-il, et sans le signaler: en effet, qu'il s'agisse du fait que le garçon s'était rasé en partie la tête et teint les cheveux (4.1) ou de la découverte des cadavres (4.3), il ne peut s'agir que de témoignages de même nature. Le narrateur $\left[\mathrm{N}^{1}\right]$ n'a pas vu l'adolescent (celui-ci était en cours d'interrogatoire) et ne saurait avoir assisté à la découverte des cadavres. Les sources d'information de l'AP sont implicitement reconnues dans (4.3): *et la police pense qu'ils ont été tuées jeudi matin. Ainsi toute la matière narrative a-t-elle la même origine: elle émane exclusivement des trois narrateurs [n] c'est-à-dire de l'institution policière, et $\left[\mathrm{N}^{2}\right]$ se contente de la redistribuer et de l'organiser. J'en vois une preuve d'ailleurs dans le fait que, dans le paragraphe en caractères gras par lequel débute le commentaire, l'accent est exclusivement mis sur l'épisode de l'arrestation, et donc sur l'intervention de la police et sur l'efficacité de celle-ci. Le choix typographique exprime une fois encore la hiérarchie des informations. Mais, dans ce même paragraphe, on remarquera que le suspect est déjà qualifié de meurtrier ("après avoir massacré *), alors que dans le paragraphe qui suit il n'est apparemment pas encore inculpé (cétait en cours d'interrogatoire [...] nous avons l'intention d'introduire une demande pour qu'il soit inculpé »).

Sur ce premier discours se superpose le discours journalistique qui se caractérise dans ce cas par:

a) la redondance de l'information considérée comme essentielle qui affecte ici les trois points suivants:

- le massacre à la hache (titre et [3D);

- le souhait que l'adolescent soit jugé comme un adulte ([3] et [4]);

- l'adolescent avait des problèmes avec son père (14]) (autrement dit: les faits, l'accusation, les preuves).

b) la recherche du sensationnel (typographie, photo, montage) aux dépens d'une authentique objectivité. 
On constate ainsi que la pratique discursive journalistique non seulement cautionne le discours policier en dissimulant certaines des traces de l'énonciation, mais encore redouble les effets sur l'imaginaire et la sensibilité du public.

Ces deux pratiques recourent à un même implicite idéologique. J'ai essayé de le montrer en évoquant le débat qui porte sur des conceptions contradictoires de l'administration de la justice. Il en est de même lorsqu'il s'agit de la représentation de l'adolescent: ‘le présumé meurtrier * se déguise, après le meurtre, en skinbead (s'était en partie rasé la tête et teint les cheveux") et à son propos se trouve évoqué un monde trouble, replié sur lui même, interdit aux adultes (*une cassette qu'il avait achetée et qu'il ne voulait pas que son père écoute •), c'est-à-dire un monde dont les interdits ne correspondent pas aux interdits des adultes.

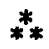

Ce court fait divers peut-être examiné à plusieurs niveaux. Sans doute ne s'agit-il pas de s'interroger sur les faits eux-mêmes (on estimera sur ce point que la culpabilité de l'adolescent n'est pas établie) mais essentiellement sur le discours produit. De ce point de vue, deux remarque sont à faire.

Au fait que, sur la photo, le fils usurpe l'autorité paternelle, répond, au niveau collectif, le désir qu'exprime le procureur d'assimiler l'adolescent à l'adulte en matière pénale. L'affrontement tout autant que les perturbations que nous avons relevées sur la photo se déplacent de la sorte de la cellule familiale au plan collectif où le représentant de l'État qui, sur le plan symbolique, devait assumer la fonction paternelle en exerçant la loi, ne fait que réclamer vengeance. Or, ce faisant, il fait surgir, dans l'ombre du présumé meurtrier, une cohorte menaçante d'adolescents perdus. Au delà des faits et quelle qu'en soit la brutalité, ce texte réactive l'angoisse de l'ancienne génération face à la montée de la nouvelle: c'est bien sur ce point que notre lecture sémiotique de la photo coincide avec notre analyse des diverses pratiques discursives redistribuées par le texte, y compris celle de l'implicite du discours social. Or cette stratégie se joue sur deux scènes parfaitement homologues: la cellule familiale considérée comme un havre de paix et une société qui est supposée être 
86

consensuelle. Choisir cette perspective permet de mieux comprendre les réactions du Procureur, car cette tragédie familiale peut être lue au niveau symbolique comme représentation fantasmatique et prémonitoire de ce qui pourrait advenir sur le plan social.

Ma deuxième remarque porte sur l'articulation des deux pratiques discursives qui opèrent ici sur l'implicite idéologique. On s'aperçoit que contrairement à ce qu'on dit communément de sa fonction, le discours journalistique amplifie les effets pervers du discours policier. 